\title{
ANÁLISE DA SIMETRIA TÉRMICA EM ATLETAS DE NATAÇÃO DE ALTO RENDIMENTO
}

\section{Cristiane Mara de Rezende', Alisson Gomes da Silva ${ }^{2,3}$, Samuel Gonçalves de Almeida Encarnação ${ }^{3}$, Miguel Araújo Carneiro Júnior ${ }^{4}$, João Carlos Bouzas Marins ${ }^{5}$}

1 Centro Universitário Claretiano, Unidade Belo Horizonte

2 Seção de Educação Física, Escola Preparatória de Cadetes do Ar

${ }_{3}^{3}$ Programa de Pós-Graduação Associado em Educação Física, Departamento de Educação Física, Universidade Federal de Viçosa

Resumo: - Este estudo analisou o perfil de simetria térmica de membros superiores e inferiores de nadadores de elite. Foram obtidos termogramas de 27 nadadores, sendo nove mulheres (idade: $18.0 \pm$ 1.0 anos, massa corporal: $58.9 \pm 5.4 \mathrm{~kg}$, estatura: $168.0 \pm 2.1 \mathrm{~cm}$ ) e 18 homens (idade: $19.5 \pm 1.2$ anos, massa corporal: $73.7 \pm 6.3 \mathrm{~kg}$, estatura: $174.6 \pm 6.8 \mathrm{~cm}$ ). A temperatura irradiada da pele (TIP) foi mensurada nas mãos, antebraços, braços, coxas, pernas e ombros. 0 teste $T$ independente indicou similaridade térmica entre os lados direito e esquerdo em todas regiões corporais analisadas ( $p>0.05)$, tanto em homens quanto em mulheres. As diferenças térmicas bilaterais médias foram consideradas clinicamente aceitáveis em todas regiões corporais $\left(\leq 0.49^{\circ} \mathrm{C}\right)$, com tamanhos de efeito variando entre insignificante e pequeno. Em conclusão, nadadores de alto rendimento apresentaram simetria térmica contralateral nos membros superiores e inferiores, sugerindo uma avaliação termográfica normal indicativa de ausência de lesão.

Palavras-chave: natação; temperatura da pele; termorregulação; termografia.

Abstract: This study analyzed the thermal symmetry profile of upper and lower limbs in high-performance swimmers. Thermograms were obtained from 27 swimmers, being nine females (age: $18.0 \pm 1.0$ years old, body mass: $58.9 \pm 5.4 \mathrm{~kg}$, height: $168.0 \pm 2.1 \mathrm{~cm}$ ) and 18 males (age: $19.5 \pm 1.2$ years old, body mass: $73.7 \pm 6.3 \mathrm{~kg}$, height: $174.6 \pm 6.8 \mathrm{~cm}$ ). Skin temperatures were measured in the hands, forearms, arms, thighs, legs, and shoulders. Independent T-test indicated thermal similarity between the right and left sides in all analyzed body regions $(p>0.05)$, both in males and females. In all body regions, mean bilateral thermal differences were considered clinically acceptable $\left(\leq 0.49^{\circ} \mathrm{C}\right)$, with effect sizes ranging between insignificant and small. In conclusion, the high-performance swimmers showed contralateral thermal symmetry in upper and lower limbs, which suggests a normal thermographic assessment indicative of the absence of injuries.

Keywords: swimming; skin temperature; thermoregulation; thermography. 


\section{INTRODUÇÃO}

A alta demanda psicofisiológica imposta a atletas de alto rendimento pode desencadear um quadro de sobretreinamento e lesões, especialmente em períodos de desequilíbrio prolongado entre o estresse e a recuperação do treinamento. Nesse cenário, recursos tecnológicos aplicados ao esporte têm contribuído para aprimorar o monitoramento da resposta psicofisiológica e da recuperação de atletas. As informações obtidas nesse processo favorecem a realização de ajustes na prescrição do treinamento, visando potencializar o desempenho e prevenir lesões ${ }^{1}$.

A termografia infravermelha (TI) tem sido apontada como uma ferramenta valiosa na prevenção de lesões em atletas ${ }^{2,3}$. A técnica registra o calor irradiado da superfície corporal gerando imagens térmicas (termogramas), por meio das quais é possível quantificar a temperatura irradiada da pele (TIP) de maneira não invasiva, segura e sem contato ${ }^{4,5}$. O monitoramento regular da TIP permite estabelecer o mapa térmico de um atleta considerando tanto as diferenças térmicas bilaterais quanto as variações térmicas das extremidades corporais frente às regiões centrais do corpo, que variam conforme indicado em estudos prévios realizados com sujeitos adultos saudáveis ${ }^{6}$ e militares ${ }^{7,8}$. Além disso, é possível obter informações sobre possíveis alterações fisiológicas que podem alterar a temperatura de apenas um segmento, gerando assimetria, ou de ambos os lados simultaneamente ${ }^{3,5}$.

É sabido que, em condições normais, a TIP é similar entre regiões corporais contralaterais ${ }^{8,9}$. Em contrapartida, diferenças bilaterais maiores que $0.7^{\circ} \mathrm{C}$ podem estar associadas a quadros de anormalidades fisiológicas ou estruturais em atletas ${ }^{3,4}$. Outros autores sugerem que diferenças térmicas bilaterais $\leq 0.49{ }^{\circ} \mathrm{C}$ são normais, enquanto assimetrias de maior magnitude demandam reavaliações $\left(0.5\right.$ a $\left.0.79{ }^{\circ} \mathrm{C}\right)$, a adoção de medidas preventivas (0.8 a 1.0 $\left.{ }^{\circ} \mathrm{C}\right)$ ou até mesmo a suspensão temporária do treinamento $\left(>1^{\circ} \mathrm{C}\right)$ para avaliação médica ${ }^{5}$. Desse modo, a TI pode ser uma ferramenta importante para prevenção de lesões em atletas quando diferenças térmicas bilaterais consideráveis são identificadas.

Para facilitar a interpretação de dados termográficos, é necessário estabelecer o perfil de normalidade em diferentes populações sem patologia e/ou lesão. Alguns estudos estabeleceram o perfil térmico de jogadores de futebol ${ }^{10,11}$. Contudo, pelo nosso conhecimento, nenhum estudo caracterizou o perfil termográfico de nadadores de alto rendimento. Foram identificados trabalhos prévios apenas com nadadores recreativos universitários ${ }^{12,13}$, que possuem volume e intensidade de treinamento diferentes de nadadores de elite. A construção de dados normativos pode ajudar a estabelecer padrões de referência em diferentes regiões corporais de interesse (RCI), com foco nas áreas mais afetadas por lesões, como os ombros de nadadores ${ }^{14,15}$, permitindo identificar condições de hiper ou hiporradiação cutânea e adotar medidas preventivas.

A natação, por ser um esporte cíclico, demanda uma repetição elevada de movimentos com carga similar em ambos dimídios corporais. Isso implica que as respostas fisiológicas e adaptações musculoesqueléticas bilaterais devam ser semelhantes. Treinos de natação, especialmente os de elite, possuem volumes elevados (6000 - 10000 metros por dia) ${ }^{16}$, o que impõe elevadas cargas de movimentos repetitivos em várias articulações, podendo provocar problemas como bursite e tendinites ${ }^{14,15}$, o que pode alterar a TIP e ser detectado pela TI. Ter um referencial de perfil térmico de nadadores de elite pode ajudar a estabelecer este padrão, 
além de monitorar o estado de normalidade do atleta desta modalidade, colaborando assim com os profissionais envolvidos para um melhor gerenciamento da carga de treino. Assim, o objetivo do presente estudo foi analisar o perfil de simetria térmica de membros superiores e inferiores de nadadores de alto rendimento.

\section{Metodologia}

Este estudo transversal analisou 27 nadadores de alto rendimento, sendo nove do sexo feminino (idade: $18.3 \pm 1.0$ anos, massa corporal: $58.9 \pm 5.4 \mathrm{~kg}$ e estatura: $168.0 \pm 2.2 \mathrm{~cm}$ ) e 18 masculino (idade: $19.5 \pm 1.2$ anos, massa corporal: $73.7 \pm$ $6.3 \mathrm{~kg}$ e estatura: $174.6 \pm 6.8 \mathrm{~cm}$ ). Os voluntários competiam em nível nacional e internacional, vinculados a uma equipe de natação de Belo Horizonte - MG, Brasil. Todos realizavam treinamento sistematizado seis vezes por semana (segunda a sábado, 10 treinos semanais), 120 minutos por sessão, duas vezes ao dia, exceto na quarta-feira e sábado, quando o treinamento ocorria apenas de manhã. No momento da coleta, eles encontravam-se no período de transição da fase básica para a fase específica do macrociclo de treinamento. Como a TIP sofre interferência de diversos fatores ${ }^{17}$, os seguintes critérios de exclusão foram adotados: ter realizado fisioterapia no dia ou na véspera da coleta; sintomas de dor em qualquer região corporal; ser fumante; distúrbios de sono; febre nos últimos sete dias; ter consumido medicamento anti-inflamatório ou antitérmico no período de duas semanas antes ou durante as avaliações; ou lesão musculoesquelética recente.

Os atletas foram esclarecidos sobre a pesquisa e deram o consentimento por escrito para participarem voluntariamente do estudo. 0 estudo seguiu os critérios da legislação brasileira de acordo com as normas da Resolução 466/2012 do Conselho Nacional de Saúde.
A coleta de termogramas seguiu as recomendações da Associação Europeia de Termologia ${ }^{18}$. Os participantes foram orientados a evitar a ingestão de álcool ou cafeína, não utilizar qualquer tipo de creme ou cosmético na pele, evitar exposição solar pré-avaliação e não tomar banho $1 \mathrm{~h}$ antes da avaliação. Os termogramas foram obtidos entre $15 \mathrm{~h}$ e 16h, após $48 \mathrm{~h}$ de repouso sem treino. Um período mínimo de aclimatação de 10 min foi respeitado em uma sala com temperatura entre 21 e $22^{\circ} \mathrm{C}$ para permitir a estabilização da TIP6. Durante a aclimatação, os voluntários ficaram em pé sobre uma superfície de madeira, vestindo sunga ou biquíni, e foram orientados a não cruzarem os braços, não realizarem movimentos bruscos e não esfregarem as mãos ou qualquer outra parte do corpo.

Após aclimatação, seis termogramas por avaliado foram obtidos com um termovisor TIR-25 (Fluke®, Everett, EUA), com amplitude de medição de $-20 \mathrm{a}+350^{\circ} \mathrm{C}$, precisão de $\pm 2 \%$, sensibilidade $\leq 0.1{ }^{\circ} \mathrm{C}$, banda de espectral dos infravermelhos de $7.5 \mu \mathrm{m}$ a $14 \mu \mathrm{m}$, taxa de atualização de 9 $\mathrm{Hz}$ e Sistema FPA (Focal Plane Array) de $160 \times 120$ pixels. A câmera foi ligada 15 min antes para permitir a estabilização do sensor e foi posicionada a 4 metros do sujeito, perpendicular às regiões corporais de interesse (RCI). A emissividade foi configurada em $0.98^{19}$.

A TIP média foi quantificada nas RCls dos braços, antebraços, mãos, ombros, coxas e pernas, as quais foram delimitadas manualmente no software SmartView 3.1 (Fluke®, Everett, EUA). Pontos anatômicos utilizados previamente em outros estudos ${ }^{6,20}$ foram adotados para guiar a delimitação das RCls, a saber: a) mão: junção do $3^{\circ}$ metacarpo com a $3^{a}$ falange proximal e processo estiloide da ulna; b) antebraço: $1^{\circ}$ terço distal do antebraço e fossa cubital; c) braço: fossa cubital e li- 
nha axilar; d) ombro: acrômio da escápula e tuberosidade deltoidea; e) coxa: $5 \mathrm{~cm}$ da borda superior da patela e borda inguinal; f) perna: $5 \mathrm{~cm}$ abaixo da borda inferior da patela e $10 \mathrm{~cm}$ acima do maléolo. A Figura 1 apresenta termogramas de um atleta avaliado com as RCls delimitadas.

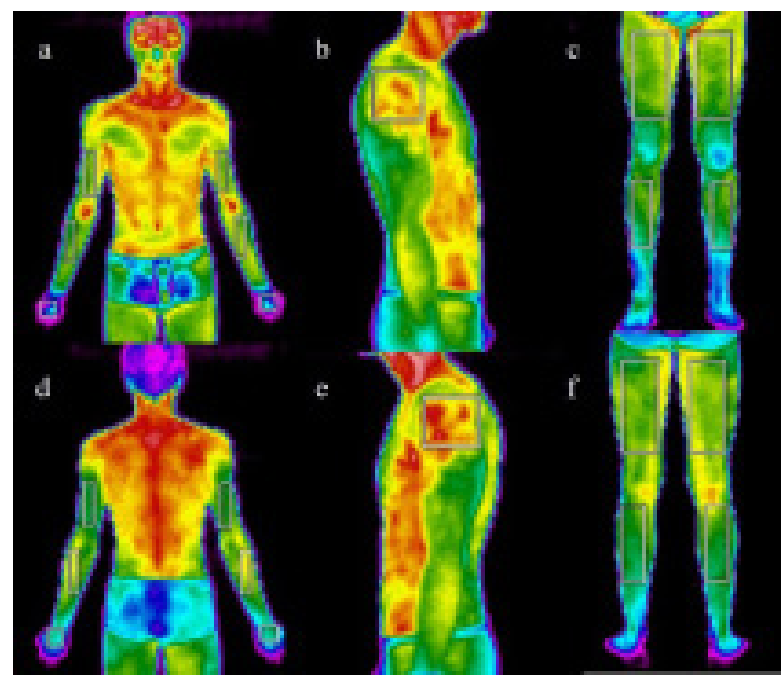

Figura 1 - Representação dos termogramas obtidos e regiões corporais analisadas.

Nota: braço, antebraço e mão na visão anterior (a) e posterior (d); ombro na visão lateral $(b, e)$; coxa e perna na visão anterior (c) e posterior (f).

Os valores de TIP média foram considerados para a análise. Nós utilizamos estatística descritiva incluindo média $(M)$ e desvio padrão $(D P)$ para apresentar os dados. As diferenças térmicas bilaterais de cada $\mathrm{RCI}$ foram calculadas subtraindo a média de TIP do lado direito pela média de TIP do lado esquerdo.

Como o pressuposto de normalidade foi constatado pelo teste de Shapiro-Wilk, o teste $T$ independente foi usado para comparar a TIP entre os lados direito e esquerdo de cada $\mathrm{RCl}$, por gênero. Um nível de significância de $p \leq 0.05$ foi adotado. Além disso, o tamanho do efeito (TE) foi calculado para estimar a magnitude das diferenças com base no d de Cohen, considerando os valores $\leq 0.19,0.2-0.49,0.5$ - 0.79 e $\geq 0,8$ como os limites para efeitos insignificantes, pequenos, médios e grandes, respectivamente ${ }^{21}$.

A distribuição percentual geral das dife- renças térmicas bilaterais foi quantificada em cinco zonas de temperatura $\left(\leq 0.49^{\circ} \mathrm{C}\right.$, $0.5-0.79{ }^{\circ} \mathrm{C}, 0.8-1.0{ }^{\circ} \mathrm{C}, 1.1-1.59{ }^{\circ} \mathrm{C}$ e $\geq 1.6^{\circ} \mathrm{C}$ ) para cada RCl. O software SPSS, versão 20.0, foi usado para todas análises (SPSS Inc., Chicago, IL).

\section{RESULTADOS}

As Tabelas $\mathbf{1}$ e $\mathbf{2}$ apresentam os valores de TIP e diferença térmica bilateral de cada RCI para atletas do sexo masculino e feminino, respectivamente. Não houve diferença significativa de temperatura entre os lados direito e esquerdo em todas as RCls ( $p>0.05$ ) para ambos os gêneros. As diferenças térmicas bilaterais variaram de 0 a $0.4{ }^{\circ} \mathrm{C}$, sendo que o TE foi insignificante

Tabela 1 - Temperatura média da pele $\left({ }^{\circ} \mathrm{C}\right)$ e diferença térmica bilateral de nadadores homens.

\begin{tabular}{|c|c|c|c|c|}
\hline \multirow[b]{2}{*}{ Região corporal } & \multicolumn{4}{|c|}{ Temperatura irradiada da pele $\left({ }^{\circ} \mathrm{C}\right)$} \\
\hline & $M(D P)$ & Dif. & $p$ & TE \\
\hline \multicolumn{5}{|l|}{ Visão Anterior } \\
\hline Mão direita & $28,7(1,8)$ & \multirow{2}{*}{0,1} & \multirow{2}{*}{0,823} & \multirow{2}{*}{0,06} \\
\hline Mão esquerda & $28,8(1,7)$ & & & \\
\hline Antebraço direito & $31,5(0,6)$ & \multirow{2}{*}{0,4} & \multirow{2}{*}{0,052} & \multirow{2}{*}{0,67} \\
\hline Antebraço esquerdo & $31,9(0,6)$ & & & \\
\hline Braço direito & $32,0(0,7)$ & \multirow{2}{*}{0,4} & \multirow{2}{*}{0,095} & \multirow{2}{*}{0,53} \\
\hline Braço esquerdo & $32,4(0,8)$ & & & \\
\hline Coxa direita & $30,9(0,8)$ & \multirow{2}{*}{0,0} & \multirow{2}{*}{0,876} & \multirow{2}{*}{0} \\
\hline Coxa esquerda & $30,9(0,8)$ & & & \\
\hline Perna direita & $31,2(0,7)$ & \multirow{2}{*}{0,1} & \multirow{2}{*}{0,613} & \multirow{2}{*}{0,14} \\
\hline Perna esquerda & $31,1(0,7)$ & & & \\
\hline \multicolumn{5}{|l|}{ Visão Posterior } \\
\hline Mão direita & $28,9(1,2)$ & \multirow{2}{*}{0,2} & \multirow{2}{*}{0,702} & \multirow{2}{*}{0,15} \\
\hline Mão esquerda & $29,1(1,4)$ & & & \\
\hline Antebraço direita & $31,4(0,5)$ & \multirow{2}{*}{0,2} & \multirow{2}{*}{0,119} & \multirow{2}{*}{0,40} \\
\hline Antebraço esquerdo & $31,2(0,5)$ & & & \\
\hline Braço direito & $30,4(0,5)$ & \multirow{2}{*}{0,1} & \multirow{2}{*}{0,578} & \multirow{2}{*}{0,20} \\
\hline Braço esquerdo & $30,3(0,5)$ & & & \\
\hline Coxa direita & $31,2(0,8)$ & \multirow{2}{*}{0,2} & \multirow{2}{*}{0,435} & \multirow{2}{*}{0,23} \\
\hline Coxa esquerda & $31,0(0,9)$ & & & \\
\hline Perna direita & $30,6(0,6)$ & \multirow{2}{*}{0,2} & \multirow{2}{*}{0,379} & \\
\hline Perna esquerda & $30,4(0,8)$ & & & \\
\hline Visão Lateral & & & & \\
\hline Ombro direito & $32,6(0,8)$ & $0 ?$ & בראח & 00 \\
\hline Ombro esquerdo & $32,8(0,8)$ & & & \\
\hline
\end{tabular}

Nota. Dif. = diferença de temperatura entre os lados; * diferença significativa entre os lados ( $\leq \leq 0.05)$; TE = tamanho do efeito. 
ou pequeno para as $\mathrm{RCls}$ com assimetrias de até $0.3^{\circ} \mathrm{C}$, e moderado para assimetrias de $0.4^{\circ} \mathrm{C}$ (antebraço de homens e mulheres e braço de homens na visão anterior).

Tabela 2 - Temperatura média da pele $\left({ }^{\circ} \mathrm{C}\right)$ e diferença térmica bilateral de nadadoras.

\begin{tabular}{|c|c|c|c|c|}
\hline \multirow[b]{2}{*}{ Região Corporal } & \multicolumn{4}{|c|}{ Temperatura irradiada da pele $\left({ }^{\circ} \mathrm{C}\right)$} \\
\hline & $M(D P)$ & Dif. & $p$ & $\mathrm{TE}$ \\
\hline \multicolumn{5}{|l|}{ Visão Anterior } \\
\hline Mão direita & $27,6(1,6)$ & \multirow{2}{*}{0,1} & \multirow{2}{*}{0,900} & \multirow{2}{*}{0,07} \\
\hline Mão esquerda & $27,7(1,4)$ & & & \\
\hline Antebraço direito & $30,9(0,6)$ & \multirow{2}{*}{0,4} & \multirow{2}{*}{0,319} & \multirow{2}{*}{0,61} \\
\hline Antebraço esquerdo & $31,3(0,7)$ & & & \\
\hline Braço direito & $31,5(0,7)$ & \multirow{2}{*}{0,3} & \multirow{2}{*}{0,321} & \multirow{2}{*}{0,40} \\
\hline Braço esquerdo & $31,8(0,8)$ & & & \\
\hline Coxa direita & $30,0(0,8)$ & \multirow{2}{*}{0,2} & \multirow{2}{*}{0,761} & \multirow{2}{*}{0,23} \\
\hline Coxa esquerda & $30,2(0,9)$ & & & \\
\hline Perna direita & $30,9(1,1)$ & \multirow{2}{*}{0,2} & \multirow{2}{*}{0,748} & \multirow{2}{*}{0,18} \\
\hline Perna esquerda & $31,1(1,1)$ & & & \\
\hline \multicolumn{5}{|l|}{ Visão Posterior } \\
\hline Mão direita & $28,4(0,9)$ & \multirow{2}{*}{0,1} & \multirow{2}{*}{0,770} & \multirow{2}{*}{0,16} \\
\hline Mão esquerda & $28,5(1,0)$ & & & \\
\hline Antebraço direita & $31,2(0,5)$ & \multirow{2}{*}{0,2} & \multirow{2}{*}{0,421} & \multirow{2}{*}{0,36} \\
\hline Antebraço esquerdo & $31,0(0,6)$ & & & \\
\hline Braço direito & $30,0(0,7)$ & \multirow{2}{*}{0,2} & \multirow{2}{*}{0,287} & \multirow{2}{*}{0,25} \\
\hline Braço esquerdo & $29,8(0,9)$ & & & \\
\hline Coxa direita & $30,4(0,8)$ & \multirow{2}{*}{0,2} & \multirow{2}{*}{0,643} & \multirow{2}{*}{0,25} \\
\hline Coxa esquerda & $30,2(0,8)$ & & & \\
\hline Perna direita & $30,4(0,7)$ & \multirow{2}{*}{0,1} & \multirow{2}{*}{0,665} & 011 \\
\hline Perna esquerda & $30,3(0,7)$ & & & \\
\hline Visão Lateral & & & & \\
\hline Ombro direito & $32,1(0,7)$ & ก? & 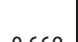 & ? \\
\hline Ombro esquerdo & $32,3(1,0)$ & 0,2 & 0,002 & $0,<0$ \\
\hline
\end{tabular}

Nota. Dif. = diferença de temperatura entre os lados; * diferença significativa entre os lados ( $p \leq 0.05) ;$ TE = tamanho do efeito.

A Tabela 3 apresenta a distribuição percentual de diferença térmica bilateral em diferentes zonas de temperatura. Em todas as RCls, a maior parte das diferenças bilaterais se concentraram em uma zona de temperatura $\leq 0.49^{\circ} \mathrm{C}$, seguida pela faixa de 0.5 a $0.79^{\circ} \mathrm{C}$. Apenas casos isolados de assimetrias elevadas foram verificados nas zonas de 0.8 a $1.0{ }^{\circ} \mathrm{C}$ e 1.1 a $1.59{ }^{\circ} \mathrm{C}$. Não houve assimetria térmica $>1.6^{\circ} \mathrm{C}$ em qualquer $\mathrm{RCl}$.
Tabela 3 - Distribuição percentual de diferença térmica bilateral $\left({ }^{\circ} \mathrm{C}\right)$ da amostra por magnitude de assimetria.

\begin{tabular}{|r|c|c|c|c|}
\hline & \multicolumn{4}{|c|}{ Zona de Temperatura ( $\left.{ }^{\circ} \mathrm{C}\right)$} \\
\hline Região corporal & $\leq 0.49$ & $0.5-0.79$ & $0.8-1.0$ & $1.1-1.59$ \\
\hline Visão Anterior & \multicolumn{5}{|c|}{} \\
\hline Mão & $63 \%$ & $25 \%$ & $8 \%$ & $4 \%$ \\
\hline Antebraço & $63 \%$ & $33 \%$ & $0 \%$ & $4 \%$ \\
\hline Braço & $44 \%$ & $48 \%$ & $4 \%$ & $4 \%$ \\
\hline Coxa & $78 \%$ & $22 \%$ & $0 \%$ & $0 \%$ \\
\hline Perna & $74 \%$ & $26 \%$ & $0 \%$ & $0 \%$ \\
\hline Visão Posterior & & & & \\
\hline Mão & $57 \%$ & $23 \%$ & $12 \%$ & $8 \%$ \\
\hline Antebraço & $77 \%$ & $11 \%$ & $8 \%$ & $4 \%$ \\
\hline Braço & $89 \%$ & $0 \%$ & $11 \%$ & $0 \%$ \\
\hline Coxa & $74 \%$ & $26 \%$ & $0 \%$ & $0 \%$ \\
\hline Perna & $55 \%$ & $26 \%$ & $15 \%$ & $4 \%$ \\
\hline Visão Lateral & \multicolumn{5}{|c|}{} \\
\hline Ombro & $60 \%$ & $12 \%$ & $15 \%$ & $13 \%$ \\
\hline & \multicolumn{5}{|c|}{} \\
\hline
\end{tabular}

\section{DIscussão}

O principal achado deste estudo foi a identificação de simetria de TIP entre RCls bilaterais em nadadores de alto rendimento (Tabelas 1 e 2). Além disso, as diferenças térmicas bilaterais foram aceitáveis do ponto de vista clínico, já que elas foram classificadas, em maioria, como insignificantes ou pequenas com base no cálculo do TE. Nossos resultados, portanto, apontam para um equilíbrio térmico nas $\mathrm{RCl}$ estudadas e estão de acordo com trabalhos prévios que reportaram níveis similares de simetria térmica em nadadores recreativos universitários ${ }^{12,13}$. Isto demonstra, também no alto rendimento, a distribuição da carga física de forma igualitária entre dimídios corporais desta modalidade cíclica.

Considerando que nenhum atleta foi diagnosticado com lesão, os resultados indicam que, em condições normais, e como um padrão de referência para avaliação termográfica de nadadores de alto rendimento, deve haver simetria térmica entre RCls contralaterais. A faixa normal de diferença de temperatura entre uma $\mathrm{RCl}$ e sua região contralateral em indiví- 
duos normais e em atletas ainda não foi estabelecida. Alguns autores encontraram diferentes valores em população de não atletas, chegando a $0.3{ }^{\circ} \mathrm{C}^{20,22}, 0.4{ }^{\circ} \mathrm{C}^{23}$, ou $0.5{ }^{\circ} \mathrm{C}^{24,25}$. Em atletas, Hildebrandt et al. ${ }^{3}$ estabeleceram um valor de $0.7{ }^{\circ} \mathrm{C}$ como o limite aceitável de assimetria, enquanto, mais recentemente, Marins et al. ${ }^{5}$ classificaram como normais assimetrias de até $0.49^{\circ} \mathrm{C}$.

Nesse sentido, os resultados de nosso estudo mostram valores médios de assimetria condizentes com os apresentados na literatura. Entretanto, mais trabalhos são necessários para estabelecer a faixa de normalidade de assimetria térmica em atletas, especialmente na natação, uma vez que trabalhos prévios foram realizados apenas com nadadores recreativos universitários ${ }^{12,13}$. Estudos realizados com outras modalidades reportaram um perfil de simetria térmica de membros inferiores $\leq 0.2{ }^{\circ} \mathrm{C}$ em futebolistas de categoria de base e profissionais ${ }^{10,11}$. Vale ressaltar que o perfil térmico pode ser específico em função da modalidade, como foi demonstrado por Estal et al. ${ }^{26}$, que encontraram algumas assimetrias nas pernas de lutadores de Muay Thay, em função da característica acíclica desse esporte. Nesse caso, a assimetria pode ser considerada como uma característica do perfil térmico da modalidade, sem associação com risco de lesão.

Com relação aos valores absolutos de TIP média verificados, nós ressaltamos que eles estão de acordo com uma faixa de variação térmica normal reportada em trabalho prévio realizado com adultos saudáveis ${ }^{20}$. Dados normativos podem ajudar a interpretar dados a partir de uma avaliação para identificar se uma RCI apresenta um desequilíbrio térmico em relação a sua área contralateral, ou se a $\mathrm{RCl}$ apresenta um estado de hiper-radiação ou hiporradiação, indicando, assim, a necessidade de um acompanhamento mais detalhado para confirmar essa condição e estabelecer a causa deste comportamento térmico anormal na pele.

Há sugestões na literatura de que diferenças térmicas bilaterais maiores que 1 ${ }^{\circ} \mathrm{C}$ sejam um forte indicativo de anormalidade $^{27}$. No presente estudo, considerando os valores médios de TIP da amostra, não houve $\mathrm{RCl}$ com assimetrias maiores que $1^{\circ} \mathrm{C}$ (Tabelas 1 e 2). Além disso, a maioria das $\mathrm{RCl}$ analisadas possui uma alta concentração de assimetrias térmicas menores que $0.5^{\circ} \mathrm{C}$ (Tabela 3), o que reforça um estado de normalidade térmica e a ausência de alterações fisiológicas locais. Esse comportamento é similar ao observada em outros estudos com populações de não atletas ${ }^{20,24}$.

Ao considerar o limite de normalidade de $0.7^{\circ} \mathrm{C}$ para assimetria em atletas ${ }^{3}$, observa-se claramente que a maior parte dos nadadores avaliados se encontra em condições ideais (Tabela 3). Contudo, casos isolados de assimetrias superiores a 0.7 ${ }^{\circ} \mathrm{C}$ foram observados na região de membros superiores, com um destaque para os $13 \%(n=3)$ encontrados acima de $1^{\circ} \mathrm{C}$ na $\mathrm{RCl}$ dos ombros. Esse resultado pode ser atribuído à alta carga física aplicada nos ombros dos nadadores, o que poderia induzir uma alteração térmica nessa área. No ambiente prático, é recomendável que esses casos sejam reavaliados e, dependendo da magnitude da assimetria, sejam adotadas medidas preventivas ou até mesmo a suspensão provisória do treinamento para que a causa dessa alteração seja investigada ${ }^{5}$. Não foi possível acompanhar a evolução das assimetrias observadas no presente estudo em função do delineamento transversal.

Identificar um desequilíbrio térmico é uma ação muito importante de prevenção de lesão em atletas. Sabe-se que aumentos na TIP superiores aos valores habitu- 
ais podem ser causados por algum problema inflamatório, enquanto uma condição hiporradiante local é sugestiva de um quadro degenerativo em que ocorre uma redução do fluxo sanguíneo na área afeta$\mathrm{da}^{3,4,9}$.

Além disso, Tovin ${ }^{28}$ aponta que as causas da grande incidência de quadros dolorosos de nadadores de alto nível estão relacionados a fatores como a sobrecarga e desequilíbrio das estruturas anatômicas devido ao grande volume e intensidade de treinamento, ao uso incorreto de técnicas de nado que ocorre quando há fadiga, excesso ou falta de flexibilidade, déficit proprioceptivo, treinamento exclusivo dos músculos agonistas proporcionando desequilíbrio mioarticular. Isso pode produzir lesões do tipo luxações glenoumeral e acromioclavicular, tendinites, sub-luxações e instabilidade oculta, em que se observaria um aumento na TIP, possível de ser captado pela TI.

Assim como foi recomendada para outras modalidades desportivas ${ }^{11}$, nós sugerimos a inclusão da avaliação termográfica na rotina de treinamentos de nadadores de alto rendimento. Nesse caso, o histórico de avaliação de cada atleta permite identificar alguma anormalidade na TIP com maior precisão, uma vez que cada atleta seria comparado com seu perfil térmico habitual. Dessa forma, a avaliação termográfica pode melhorar o controle do treinamento, reduzir o número de lesões e economizar recursos decorrentes do tratamento, além de reduzir os afastamentos de atletas durante treinamentos e competições ao longo da temporada. Um exemplo disso foi um estudo realizado com futebolistas de categoria sub-20 que mostrou que o uso da termografia no mapeamento de assimetrias reduziu o número de lesões de 23 $(33.3 \%)$ para $14(20.3 \%)(p<0.05)$ durante um ano de acompanhamento, comparando com a temporada sem monitoramento termográfico ${ }^{29}$. Similarmente, Côrte et al. ${ }^{30}$ reduziram em $64 \%$ a incidência de lesões na temporada de uma equipe profissional de futebol em comparação à temporada anterior sem avaliação termográfica (11 versus 4 lesões). Nesse caso, os autores iniciavam um protocolo de prevenção nos futebolistas sempre que eles identificavam assimetrias térmicas maiores que 0.4 ${ }^{\circ} \mathrm{C} 48 \mathrm{~h}$ após os jogos.

As limitações do estudo foram a falta de realização de exames sanguíneos como o de creatina quinase, além de exames de imagens como ressonância magnética para analisar a relação entre casos de assimetrias de TIP superiores a $0.7{ }^{\circ} \mathrm{C}$ com algum problema físico na área. Contudo, o presente estudo proporciona informações inéditas do perfil termográfico de nadadores de alto rendimento no Brasil, que podem ser úteis como referencial para a prática profissional.

\section{ConclusÃo}

Nadadores de alto rendimento apresentaram simetria térmica contralateral com diferenças na TIP entre RCI bilaterais iguais ou menores que $0.49{ }^{\circ} \mathrm{C}$. Esse padrão simétrico sugere que o grupo de atletas apresentou uma avaliação termográfica normal. Contudo, alguns atletas apresentaram assimetrias térmicas nos ombros que demandam reavaliações, tendo em vista a importância do monitoramento individual de atletas de elite. Estudos futuros devem investigar o impacto de diferentes magnitudes de carga de treinamento na TIP e simetria térmica de nadadores em fases distintas da preparação esportiva.

\section{AgRADECIMENTOS}

Fundação de Amparo à Pesquisa do Estado de Minas Gerais (FAPEMIG). 


\section{REFERÊNCIAS}

1. Halson SL. Monitoring training load classification to understand fatigue in athletes. Sports Med. 2014; 44(2):139-147. doi: 10.1007/s40279-014-0253-z

2. Viegas F, Mello MT, Rodrigues SA., Costa CMA, Freitas LSN, Rodrigues EL, et al. The use of thermography and its control variables: a systematic review. Rev Bras Med Esporte. 2020; 26(1):82-86. doi: 10.1590/1517869220202601217833 .

3. Hildebrandt C, Raschner C, Ammer K. An overview of recent application of medical infrared thermography in sports medicine in Austria. Sensors. 2010; 10(5):47004715. doi: $10.3390 /$ s100504700

4. Bach AJE, Stewart IB, Minett GM, Costello JT. Does the technique employed for skin temperature assessment alter outcomes? A systematic review. Physiol Meas. 2015; 36(9):27-51. doi: 10.1088/0967-3334/36/9/R27

5. Marins JCB, Fernández-Cuevas I, Arnaiz-Lastras J, Fernandes AA, Sillero-Quintana M. Aplicaciones de la termografía infrarroja en el deporte: Una revisión. Rev Int Med Cien Act Fís Deporte. 2015; 15(60):805-824

6. Marins JCB, Moreira DG, Cano SP, Sillero-Quintana M, Soares DS, Fernandes AA, et al. Time required to stabilize thermographic images at rest. Infrared Phys Technol. 2014; 65:30-35. doi: 10.1016/j.infrared.2014.02.008

7. Costa CMA, Moreira DG, Sillero-Quintana M, Brito CJ, Azambuja PG, Fernandes AA, et al. Daily rhythm of skin temperature of women evaluated by infrared thermal imaging. J Therm Biol. 2018; 72:1-9. doi: 10.1016/j. jtherbio.2017.12.002

8. Costa CMA, Sillero-Quintana M, Cano SP. Daily oscillations of skin temperature in military personnel using thermography. J Roy Army Med Corps. 2016; 162(5):335-342. doi: 10.1136/jramc-2015-000462

9. Brioschi ML, Macedo JF, Macedo, RAC. Termometria cutânea: novos conceitos. J Vasc Br. 2003; 2(2):151-160.

10. Maior AS, Leporace G, Tannure M, Marocolo M. Profile of infrared thermography in elite soccer players. Motriz: J Phys Ed. 2017; 23(2):e101654. doi: 10.1590/ s1980-6574201700020013

11. Marins JCB, Fernandes A, Moreira DG, Silva FS, Costa CMA, Pimenta EM, Sillero-Quintana M. Thermographic profile of soccer players' lower limbs. Rev Andal Med Deporte. 2014; 7(1):1-6. doi: 10.1016/S18887546(14)70053-X

12. Novotny J, Ryborava S, Zacha D, Novotny Jr J, Bernacikova M, Ramadan WA. Thermographic evaluation of muscle activity after front crawl swimming in young men. Acta Bioeng Biomech. 2017; 19(4):109-116. doi: 10.5277/ABB-00924-2017-03

13. Novotny J, Rybarova S, Zacha D, Novotny Jr $J$, Bernacikova M, Ramadan WA. The influence of breaststroke swimming on the muscle activity of young men in thermographic imaging. Acta Bioeng Biomech. 2015; 17(2):121-129. doi: 10.5277/ABB-00105-2014-03

14. Martino I, Rodeo SA. The swimmer's shoulder: multidirectional instability. Curr Rev Musculoskelet Med. 2018; 11(2):167-171. doi: 10.1007/s12178-018-9485-0

15. Matzkin E, Suslavich K, Wes D. Swimmer's shoulder: painful shoulder in the competitive swimmer. J Am Acad Orthop Surg. 2016; 24(8):527-536. doi: 10.5435/ JAAOS-D-15-00313

16. Heinlein SA, Cosgarea AJ. Biomechanical considerations in the competitive swimmer's shoulder. Sports Health. 2010; 2(6):519-525. doi: $10.1177 / 1941738110377611$

17. Fernández-Cuevas I, Marins JCB, Lastras JA, Carmona PMG, Cano SP, García-Concepcíon MA, SilleroQuintana M. Classification of factors influencing the use of infrared thermography in humans: a review. Infrared Phys Technol. 2015; 71:28-55. doi: 10.1016/j. infrared.2015.02.007

18. Ammer K, Ring EF. Standard Procedures for Infrared Imaging in Medicine. In: Bronzino JD (Ed), Medical Devices and Systems. Boca Raton (FL): CRC Press; 2006. p. 111124. doi: 10.1201/9781420003864.ch36

19. Lahiri BB, Bagavathiappan S, Jayakumar T, Philip J. Medical applications of infrared thermography: a review. Infrared Phys Technol. 2012; 55:221-235. doi: 10.1016/j. infrared.2012.03.007

20. Marins JCB, Fernandes AA, Cano SP, Moreira DG, Silva FS, Costa CMA, et al. Thermal body patterns for healthy brazilian adults (male and female). J Therm Biol. 2014; 42:1-8. doi: 10.1016/j.jtherbio.2014.02.020

21. Cohen J. Statistical power analysis for the behavioral sciences. 2nd ed. New York: Routledge; 2013.

22. Brioschi ML, Cherem JA, Ruiz RC, Sardá Jr JJ, Silva FMRM. 0 uso da termografia infravermelha na avaliação do retorno ao trabalho em programa de reabilitação ampliado (PRA). Acta Fisiátr. 2009; 16(2):87-92.

23. Pichot C. Aplicación de la termografía en el dolor lumbar crónico. Rev de la Soc Española del Dolor; 2001; $8(2): 43-47$

24. Niu HH, Lui PW, Hu JS, Ting CK, Yin YC, Lo YL, et al. Thermal symmetry of skin temperature: normative data of normal subjects in Taiwan. Zhonghua Yi Xue Za Zhi (Taipei). 2001; 64(8):459-468.

25. Uematsu S, Edwin DH, Jankel WR, Kozikowski J, Trattner M. Quantification of termal asymmetry. J Neurosurg. 1988; 69:552-555. doi: 10.3171/ jns.1988.69.4.0552

26. Estal A, Brito CJ, Galindo VE, Durana ALD, Franchini E, Sillero-Quintana M. Thermal asymmetries in striking combat sports athletes measured by infrared thermography. Sci Sport. 2017; 32(2):61-67. doi: 
10.1016/j.scispo.2016.09.005

27. Ben-Eliyahu DJ. Infrared thermographic imaging in the detection of sympathetic dysfunction in patients with patellofemoral pain. J Manip Physiol Ther. 1992; 15(3):164-170.

28. Tovin BJ. Prevention and treatment of swimmer's shoulder. N Am J Sports Phys Ther. 2006;1(4):166-175.

29. Menezes P, Rhea MR, Herdy C, Simão R. Effects of strength training program and infrared thermography in soccer athletes injuries. Sports (Basel). 2018; 6(4):148. doi: $10.3390 /$ sports 6040148

30. Côrte AC, Pedrinelli A, Marttos A, Souza IFG, Grava $J$, Hernandez AJ. Infrared thermography study as a complementary method of screening and prevention of muscle injuries: pilot study. BMJ Open Sport Exerc Med. 2019; 5(1):87-92. doi: 10.1136/bmjsem-2018-000431 